\title{
Images
}

\section{Bulletproof heart: Gunshot wound to the ventricular septum without pericardial effusion or hemodynamic collapse}

\section{Lora Alkhawam, MD*; Stephen J. Greene, $\mathrm{MD}^{\dagger}$}

A 17-year-old man presented to the trauma resuscitation bay with a gunshot to the left flank, complaining only of chest tightness. A physical exam was notable for a heart rate of 72 beats/minute, blood pressure of $161 / 89 \mathrm{~mm} \mathrm{Hg}$, oxygen saturation of $100 \%$ on room air, and a single gunshot wound to the left thoracoabdomen in the anterior axillary line. Portable chest radiographs demonstrated a missile projecting over the middle of the heart (Figure 1). An emergent bedside echocardiogram noted normal biventricular function, no pericardial effusion, no hemothorax, and an immobile bullet lodged near the interventricular septum, with no color-flow Doppler

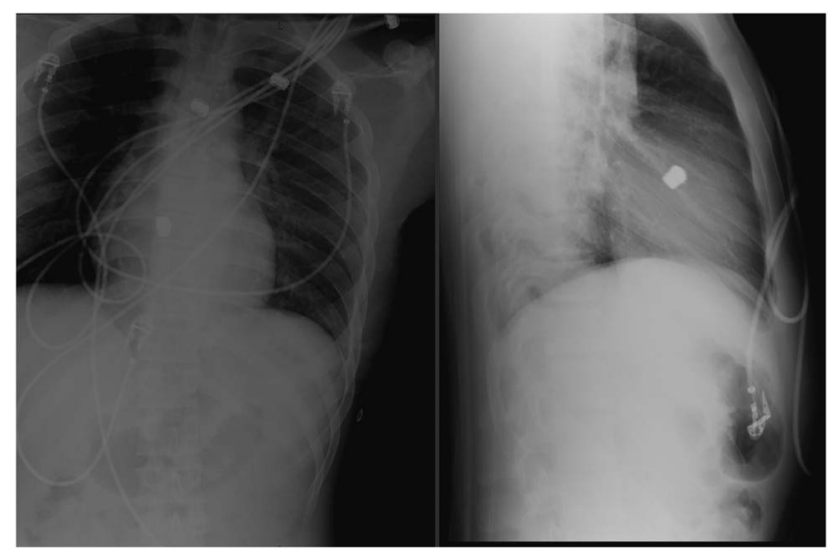

Figure 1. Portable chest radiographs showing a bullet projecting over the middle of the heart. evidence of septal defect. Initial labs showed a troponin I of $9.28 \mathrm{ng} / \mathrm{dL}$. Given apparent hemodynamic stability, the patient did not proceed emergently to the operating room. A computed tomography angiography of the chest was performed and was most notable for splenic injury and a bullet within the interventricular septum with no hemothorax, pericardial effusion, or obvious cardiac injury. The patient underwent exploratory laparotomy notable for splenic injury leading to a splenectomy and repairs of injuries to the left lateral diaphragm and left lateral lobe of the liver. The following day, the patient returned to the operating room for median sternotomy and right atriotomy where the bullet was found lodged in the membranous septum beneath the septal leaflet of the tricuspid valve. There were no atrial or ventricular septal defects and no hemothorax. The bullet was removed, and a pericardial patch repair of the perimembranous bullet cavity was performed. A $2-\mathrm{cm} \times 1.5-\mathrm{cm}$ hole in the posterior left ventricular wall with adherent clot was also noted intraoperatively and repaired. The patient recovered in the surgical intensive care unit and was discharged home in excellent condition on hospital day 15 .

Competing interests: None declared.

Keywords: trauma, gunshot wound, cardiac, cardiothoracic surgery

From the *Department of Emergency Medicine, Duke Regional Hospital, Durham, NC; and tDuke Clinical Research Institute and Division of Cardiology, Duke University Medical Center, Durham, NC.

Correspondence to: Dr. Stephen J. Greene, Duke Clinical Research Institute and Division of Cardiology, Duke University Medical Center, 2301 Erwin Road, Suite 7400, Durham, NC 27705; Email: stephen.greene@duke.edu 